\title{
Direction Finding for Bistatic MIMO Radar with Uniform Circular Array
}

\author{
Cao Yunhe, Zhang Zijing, Wang Shenghua, and Dai Fengzhou \\ National Laboratory of Radar Signal Processing, Xidian University, Xi'an 710071, China \\ Correspondence should be addressed to Cao Yunhe; cyh_xidian@163.com
}

Received 11 March 2013; Accepted 23 September 2013

Academic Editor: Ulrich Nickel

Copyright ( 2013 Cao Yunhe et al. This is an open access article distributed under the Creative Commons Attribution License, which permits unrestricted use, distribution, and reproduction in any medium, provided the original work is properly cited.

\begin{abstract}
A method of direction of arrival (DOA) and direction of departure (DOD) angle estimation based on polynomial rooting for bistatic multiple-input multiple-output (MIMO) radar with uniform circular array (UCA) configuration is proposed in this paper. The steering vector of the UCA is firstly transformed into a steering vector with a Vandermonde structure by using the JacobiAnger expansion. Then the null-spectrum function of the MIMO radar can be written as an expression in which the transmit and receive steering vectors are decoupled. Finally, a two-step polynomial rooting is used to estimate DOA and DOD of targets instead of two-dimensional multiple signal classification (MUSIC) search method for bistatic UCA MIMO radar. The angle estimation performance of the proposed method is similar to that of the MUSIC spectral search method, but the computation burden of the proposed polynomial rooting algorithm is much lower than that of the conventional MUSIC method. The simulation results of the proposed algorithm are presented and the performances are investigated and analyzed.
\end{abstract}

\section{Introduction}

Research on multiple-input multiple-output (MIMO) radar has been growing as evidenced by an increasing body of literature [1-10]. MIMO radar is characterized by using multiple antennas to simultaneously transmit orthogonal waveforms and multiple antennas to receive the reflected signals. MIMO radar has been shown to provide a number of potential benefits as compared with conventional radar, such as enhancing angle resolution, improving parameter identifiability, and increasing flexibility for transmit beam pattern design. In particular, the problem of multitarget localization in bistatic MIMO radar has received great research interests. Many methods in bistatic MIMO radar are proposed to identify and locate multiple targets [3-9] in which both the transmit array and the receive array are uniform linear arrays (ULAs). In order to avoid angle search, estimation of signal parameters via rotational invariance techniques (ESPRIT) algorithm is applied to bistatic MIMO radar [37] by exploiting the invariance property of the transmit and receive arrays. In $[8,9]$, several algorithms based on polynomial root finding procedure are proposed to estimate DOA and DOD of targets.
Unfortunately, both the ESPRIT and the polynomial rooting method are designed for ULAs. The steering vector of the ULA is dependent on $2 \pi d \sin \theta / \lambda$ (where $d$ is the interelement spacing, $\theta$ is DOA of the source, and $\lambda$ is the signal wavelength), and, hence, DOA estimation with ULA becomes ambiguous [10] beyond the range of $180^{\circ}$ (from $-90^{\circ}$ to $\left.+90^{\circ}\right)$. The following properties of uniform circular arrays (UCAs) [11-14] make them attractive in the context of DOA estimation. UCAs can provide $360^{\circ}$ azimuthal coverage. In addition, direction patterns synthesized with UCAs can be electronically rotated in the plane of the array without significant change of beam shape. ULAs, in contrast, provide only $180^{\circ}$ coverage, and beams formed with ULAs broaden as the array is steered away from boresight. Especially, UCAs configuration is very suitable for the MIMO radar, which often transmits orthogonal waveforms in each of the transmit antennas in order to detect the whole $360^{\circ}$ in the azimuth angle simultaneously.

In order to come up with computationally efficient high-resolution DOA estimators for UCAs, the so-called beamspace transform [11-13] may be applied. It rebuilds desired Vandermonde structure for the steering vectors. Consequently, methods like root-MUSIC [15-17] and ESPRIT 
may be applied to find DOAs. In [11], Tewfik and Hong have shown that it is possible to extend the Root-MUSIC to UCA using the phase mode excitation concept. In [12], Mathews and Zoltowski proposed real beamspace MUSIC to UCA that yields reduced computational complexity and better resolution. The beamspace transform works properly only under certain conditions on the array configuration that may be difficult to satisfy in some applications [13]. These algorithms require a sufficiently large number of antenna elements to avoid spatial aliasing and mapping errors that may cause error floor and excess variance [13]. It also shows that there is a significant difference in the performance of the UCA root-MUSIC technique depending on whether an even or odd number of elements is used [14]. Manifold separation technique $[18,19]$ shows an alternative method to map the array steering vectors to a Vandermonde structured virtual array with a significantly smaller fitting error than for beamspace transform.

In this paper, direction finding for bistatic MIMO radar with UCA configuration employing polynomial rooting is presented. Transmit and receive steering vectors are firstly decomposed using the Jacobi-Anger expansion [20]. Then two-dimensional direction finding in bistatic MIMO radar is transformed into double one-dimensional direction finding procedure [9]. At last, an algorithm based on polynomial root finding to estimate DOA and DOD of targets in bistatic MIMO radar with UCA configure is proposed. The computational complexity of the proposed method is low without the requirement of costly space searching procedure.

The remainder of this paper is organized as follows. In Section 2, we describe our bistatic UCA MIMO radar scheme and the associated data model. In Section 3, the proposed bistatic UCA MIMO root finding algorithm is described. Moreover, the simulation results of the proposed algorithm are presented and the performances are investigated in Section 4. Finally, Section 5 concludes the paper.

\section{Signal Model}

Consider a narrowband bistatic MIMO radar system with $M_{t}$-element transmit antennas and $M_{r}$-element receive antennas, both of which are UCAs with radii $R_{t}$ and $R_{r}$, respectively. At the transmit site, $M_{t}$ different narrow-band pulse waveforms are emitted simultaneously, which have identical bandwidth and center frequency, but are temporally orthogonal. In each receiver, the echoes are processed for all of the transmitted pulse waveforms. It is assumed that the Doppler frequencies have almost no effect on the orthogonality of the signals. Therefore, the variety of the phase within pulses caused by Doppler frequency can be ignored. There are $P$ uncorrelated targets located at the same range cell. The directions of the $p$ th target with respect to the normal direction of transmit array and receive array denoted by transmit angle $\theta_{p}$ and receive angle $\varphi_{p}$, respectively. The output of the entire matched filters at the receivers can be expressed as [10]

$$
\mathbf{X}(n)=\mathbf{A} \mathbf{S}(n)+\mathbf{N}(n),
$$

where $\mathbf{A}=\left[\mathbf{a}\left(\theta_{1}, \varphi_{1}\right), \ldots, \mathbf{a}\left(\theta_{p}, \varphi_{p}\right), \ldots, \mathbf{a}\left(\theta_{P}, \varphi_{P}\right)\right]$ is a matrix composed of the $P$ steering vectors.

Cosider that

$$
\mathbf{a}\left(\theta_{p}, \varphi_{p}\right)=\mathbf{a}_{t}\left(\theta_{p}\right) \otimes \mathbf{a}_{r}\left(\varphi_{p}\right)
$$

is the Kronecker product of the transmit and the receive steering vectors for the $p$ th target. $\mathbf{S}(n)=\left[s_{1}(n), \ldots, s_{p}(n), \ldots\right.$, $\left.s_{P}(n)\right]^{T}, s_{p}(n)=\gamma_{p} e^{j 2 \pi f_{d p} n / f_{s}}$ with $\gamma_{p}$ and $f_{d p}$ being the reflection coefficient depending on the target radar crosssection (RCS) and the Doppler frequency of the pth target, respectively. $f_{s}$ is the pulse repeat frequency, $[\cdot]^{T}$ denotes the transpose, $\mathbf{N}(n)$ denotes a noise vector assumed to be independent and identically distributed, zero-mean complex Gaussian distribution and spatially white with covariance matrix $\sigma^{2} \mathbf{I}$, where $\sigma^{2}$ is noise variance and $\mathbf{I}$ is the identity matrix.

The array covariance matrix can be written as

$$
\mathbf{R}_{x}=E\left\{\mathbf{X}(n) \mathbf{X}^{H}(n)\right\},
$$

where $[\cdot]^{H}$ denotes Hermitian transpose and $E\{\}$ denotes the statistical expectation.

The signals and the noises are assumed to be stationary, uncorrelated random processes; substituting $\mathbf{X}(n)$ from (1) into (3), we have

$$
\begin{aligned}
\mathbf{R}_{x} & =\mathbf{A} E\left\{\mathbf{S}(n) \mathbf{S}^{H}(n)\right\} \mathbf{A}^{H}+E\left\{\mathbf{N}(n) \mathbf{N}^{H}(n)\right\} \\
& =\mathbf{A R}_{s} \mathbf{A}^{H}+\sigma^{2} \mathbf{I},
\end{aligned}
$$

where $\mathbf{R}_{s}=E\left\{\mathbf{S}(n) \mathbf{S}^{H}(n)\right\}$ is the source covariance matrix and $\sigma^{2}$ is the noise power at array sensors.

In practical situations, the exact array covariance matrix $\mathbf{R}_{x}$ is unavailable and its sample estimate

$$
\widehat{\mathbf{R}}_{x}=\frac{1}{L} \sum_{l=1}^{L} \mathbf{X}(l) \mathbf{X}^{H}(l)
$$

is used, where $L$ is the number of snapshots.

The eigenvalue decomposition of $\widehat{\mathbf{R}}_{x}$ yields [10]

$$
\widehat{\mathbf{R}}_{x}=\sum_{m=1}^{M_{t} M_{r}} \lambda_{m} \mathbf{e}_{m} \mathbf{e}_{m}^{H},
$$

where $\lambda_{1} \geq \lambda_{2} \geq \cdots \geq \lambda_{P+1} \geq \cdots \geq \lambda_{M_{t} M_{r}}$ are the eigenvalues of $\widehat{\mathbf{R}}_{x}$ and $\mathbf{e}_{m}\left(m=1 \cdots M_{t} M_{r}\right)$ are the corresponding eigenvectors. The matrices

$$
\begin{gathered}
\mathbf{E}_{s}=\left[\mathbf{e}_{1}, \ldots, \mathbf{e}_{P}\right], \\
\mathbf{E}_{n}=\left[\mathbf{e}_{P+1}, \ldots, \mathbf{e}_{M_{t} M_{r}}\right]
\end{gathered}
$$

are composed of the signal and the noise subspace eigenvectors of the array covariance matrix, respectively.

The MUSIC null-spectrum function is defined as [18]

$$
f(\theta, \varphi)=\left\|\mathbf{E}_{n}^{H} \mathbf{a}(\theta, \varphi)\right\|^{2}=\mathbf{a}^{H}(\theta, \varphi) \mathbf{E}_{n} \mathbf{E}_{n}^{H} \mathbf{a}(\theta, \varphi),
$$

where $\|\cdot\|$ denotes the vector 2-norm. The spectral MUSIC technique estimates the signal DOAs from the minima of this function by means of a two-dimensional search over $\theta$ and $\varphi$. 


\section{Direction Finding for Bistatic MIMO Radar with UCA Configuration}

The transmit and receive steering vectors of the UCAs can be denoted as [11-14]

$$
\begin{aligned}
& \mathbf{a}_{t}(\theta)=\left[e^{j k R_{t} \cos \left(\theta-\beta_{1}\right)} \cdots e^{j k R_{t} \cos \left(\theta-\beta_{m}\right)} \cdots e^{j k R_{t} \cos \left(\theta-\beta_{M_{t}}\right)}\right]^{T}, \\
& \mathbf{a}_{r}(\varphi)=\left[e^{j k R_{r} \cos \left(\varphi-\alpha_{1}\right)} \cdots e^{j k R_{r} \cos \left(\varphi-\alpha_{m}\right)} \cdots e^{j k R_{r} \cos \left(\varphi-\alpha_{M_{r}}\right)}\right]^{T},
\end{aligned}
$$

where $k=2 \pi / \lambda, \lambda$ is wavelength and $\beta_{m}=2 m \pi / M_{t}$ and $\alpha_{m}=2 m \pi / M_{r}$ are the azimuth angles of the $m$ th element of transmit and receive arrays, respectively.

By using the Jacobi-Anger expansion, we can mathematically express the $m$ th element of the transmit steering vector as $[10,20]$

$$
\begin{aligned}
e^{j k R_{t} \cos \left(\theta-\beta_{m}\right)} & =\sum_{n=-\infty}^{+\infty} j^{n} J_{n}\left(k R_{t}\right) e^{-j n \beta_{m}} e^{j n \theta} \\
& =\sum_{n=-\infty}^{+\infty}\left[\mathbf{F}_{t}\right]_{m, n} e^{j n \theta},
\end{aligned}
$$

where

$$
\left[\mathbf{F}_{t}\right]_{m, n}=j^{n} J_{n}\left(k R_{t}\right) e^{-j n \beta_{m}}
$$

is the $(m, n)$ th element of the sampling matrix $\mathbf{F}_{t}$ and $J_{n}(\cdot)$ is the Bessel function of the first kind of order $n$. Consequently, we can express the transmit steering vector by

$$
\mathbf{a}_{t}(\theta)=\mathbf{F}_{t} \mathbf{d}_{t}(\theta)
$$

The $n$th component of $\mathbf{d}_{t}(\theta)$ is

$$
\left[\mathbf{d}_{t}(\theta)\right]_{n}=e^{j n \theta} .
$$

Truncating $\mathbf{F}_{t}$ and $\mathbf{d}_{t}(\theta)$ from $-\left(N_{t}-1\right) / 2$ to $\left(N_{t}-1\right) / 2$ (suppose $N_{t}$ is odd), the transmit steering vector can be approximated as

$$
\mathbf{a}_{t}(\theta) \approx \widehat{\mathbf{F}}_{t} \widehat{\mathbf{d}}_{t}(\theta)
$$

where $\widehat{\mathbf{F}}_{t}$ is an $M_{t} \times N_{t}$ matrix and

$$
\widehat{\mathbf{d}}_{t}(\theta)=\left[e^{j\left(-\left(N_{t}-1\right) / 2\right) \theta} \cdots e^{j\left(\left(N_{t}-1\right) / 2\right) \theta}\right]^{T} .
$$

We can see that $\widehat{\mathbf{d}}_{t}(\theta)$ is a Vandermonde vector which depends on the steering angle $\theta$ and the parameter $N_{t}$. The accuracy of the approximation equation (14) increases with increasing the value of $N_{t}$.

The receive steering vector can be treated in the same way as the transmit steering vector; therefore, we obtain

$$
\mathbf{a}_{r}(\varphi) \approx \widehat{\mathbf{F}}_{r} \widehat{\mathbf{d}}_{r}(\varphi)
$$

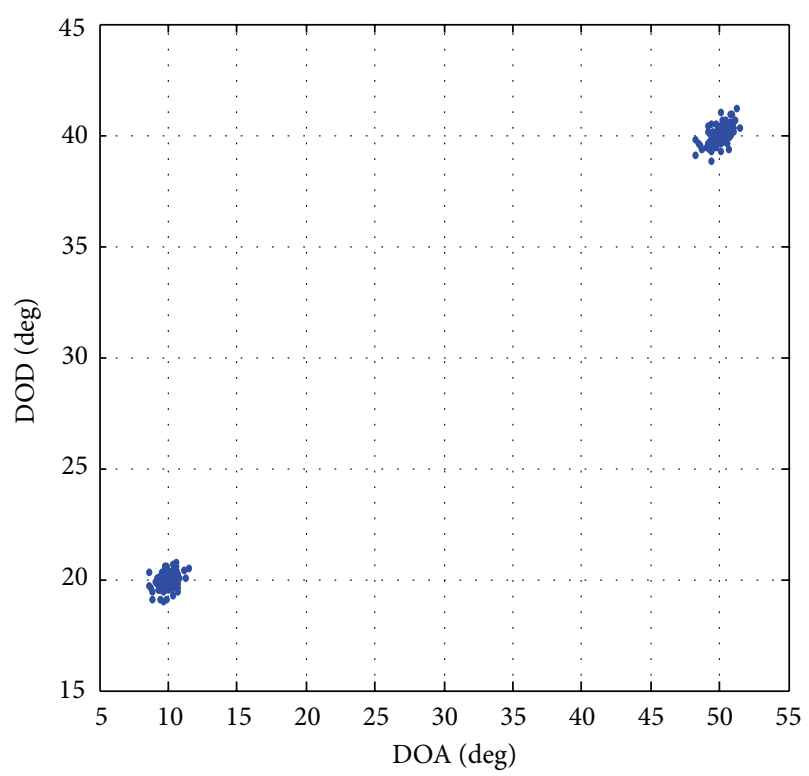

FIgURE 1: The angle estimation result of two targets.

where $\widehat{\mathbf{F}}_{r}$ is an $M_{r} \times N_{r}$ matrix and

$$
\widehat{\mathbf{d}}_{r}(\varphi)=\left[e^{j\left(-\left(N_{r}-1 / 2\right)\right) \varphi} \cdots e^{j\left(\left(N_{r}-1\right) / 2\right) \varphi}\right]^{T} .
$$

We can also see that $\widehat{\mathbf{d}}_{r}(\varphi)$ is a Vandermonde vector which depends on the steering angle $\varphi$ and the parameter $N_{r}$.

Using the notations $z_{\theta}=e^{j \theta}$ and $z_{\varphi}=e^{j \varphi},(15)$ and (17) can be written as

$$
\begin{aligned}
& \widehat{\mathbf{d}}_{t}\left(z_{\theta}\right)=\left[z^{-\left(N_{t}-1\right) / 2} \cdots z^{\left(N_{t}-1\right) / 2}\right]^{T}, \\
& \widehat{\mathbf{d}}_{r}\left(z_{\varphi}\right)=\left[z^{-\left(N_{r}-1\right) / 2} \cdots z^{\left(N_{r}-1\right) / 2}\right]^{T} .
\end{aligned}
$$

Using (2) and (16), the null-spectrum function equation (8) can be written as

$$
\begin{aligned}
f(\theta, \varphi) & =\left[\mathbf{a}_{t}(\theta) \otimes \mathbf{a}_{r}(\varphi)\right]^{H} \mathbf{E}_{n} \mathbf{E}_{n}^{H}\left[\mathbf{a}_{t}(\theta) \otimes \mathbf{a}_{r}(\varphi)\right] \\
& =\mathbf{a}_{t}^{H}(\theta) \mathbf{A}_{r}^{H} \mathbf{E}_{n} \mathbf{E}_{n}^{H} \mathbf{A}_{r} \mathbf{a}_{t}(\theta) \\
& =\mathbf{a}_{t}^{H}(\theta)\left[\Omega \mathbf{D}_{r}\left(z_{\varphi}\right)\right]^{H} \mathbf{E}_{n} \mathbf{E}_{n}^{H}\left[\Omega \mathbf{D}_{r}\left(z_{\varphi}\right)\right] \mathbf{a}_{t}(\theta) \\
& =\mathbf{a}_{t}^{H}(\theta) \mathbf{D}_{r}^{H}\left(z_{\varphi}\right) \mathbf{\Omega}^{H} \mathbf{E}_{n} \mathbf{E}_{n}^{H} \mathbf{\Omega} \mathbf{D}_{r}\left(z_{\varphi}\right) \mathbf{a}_{t}(\theta) \\
& =\mathbf{a}_{t}^{H}(\theta) \mathbf{G}\left(z_{\varphi}\right) \mathbf{a}_{t}(\theta),
\end{aligned}
$$


where

$$
\begin{gathered}
A_{r}=\left[\begin{array}{cccc}
\mathbf{a}_{r}(\varphi) & \mathbf{0}_{M_{r} \times 1} & \cdots & \mathbf{0}_{M_{r} \times 1} \\
\mathbf{0}_{M_{r} \times 1} & \mathbf{a}_{r}(\varphi) & \cdots & \mathbf{0}_{M_{r} \times 1} \\
\vdots & \vdots & \vdots & \vdots \\
\mathbf{0}_{M_{r} \times 1} & \mathbf{0}_{M_{r} \times 1} & \cdots & \mathbf{a}_{r}(\varphi)
\end{array}\right]_{M_{r} M_{t} \times M_{t}} \\
\mathbf{G}\left(z_{\varphi}\right)=\mathbf{D}_{r}^{H}\left(z_{\varphi}\right) \mathbf{\Omega}^{H} \mathbf{E}_{n} \mathbf{E}_{n}^{H} \mathbf{\Omega}_{r}\left(z_{\varphi}\right), \\
\mathbf{\Omega}=\left[\begin{array}{cccc}
\widehat{\mathbf{F}}_{r} & \mathbf{0}_{M_{r} \times N_{r}} & \cdots & \mathbf{0}_{M_{r} \times N_{r}} \\
\mathbf{0}_{M_{r} \times N_{r}} & \widehat{\mathbf{F}}_{r} & \cdots & \mathbf{0}_{M_{r} \times N_{r}} \\
\vdots & \vdots & \vdots & \vdots \\
\mathbf{0}_{M_{r} \times N_{r}} & \mathbf{0}_{M_{r} \times N_{r}} & \cdots & \widehat{\mathbf{F}}_{r}
\end{array}\right]_{M_{r} M_{t} \times N_{r} M_{t}} \\
\mathbf{D}_{r}\left(z_{\varphi}\right)=\left[\begin{array}{cccc}
\widehat{\mathbf{d}}_{r}\left(z_{\varphi}\right) & \mathbf{0}_{N_{r} \times 1} & \cdots & \mathbf{0}_{N_{r} \times 1} \\
\mathbf{0}_{N_{r} \times 1} & \widehat{\mathbf{d}}_{r}\left(z_{\varphi}\right) & \cdots & \mathbf{0}_{N_{r} \times 1} \\
\vdots & \vdots & \vdots & \vdots \\
\mathbf{0}_{N_{r} \times 1} & \mathbf{0}_{N_{r} \times 1} & \cdots & \widehat{\mathbf{d}}_{r}\left(z_{\varphi}\right)
\end{array}\right]_{N_{r} M_{t} \times M_{t}}
\end{gathered}
$$

Therefore, to solve the set in expression (19), we can first find $z_{\varphi}$ satisfying [9]

$$
\operatorname{det}\left[\mathbf{G}\left(z_{\varphi}\right)\right]=\operatorname{det}\left[\mathbf{D}_{r}^{H}\left(z_{\varphi}\right) \mathbf{\Omega}^{H} \mathbf{E}_{n} \mathbf{E}_{n}^{H} \mathbf{\Omega} \mathbf{D}_{r}\left(z_{\varphi}\right)\right]=0 .
$$

Suppose $\mathbf{B}=\boldsymbol{\Omega}^{H} \mathbf{E}_{n} \mathbf{E}_{n}^{H} \boldsymbol{\Omega}$. Obviously matrix B is an $N_{r} M_{t} \times$ $N_{r} M_{t}$ matrix that can be written as

$$
\mathbf{B}=\left[\begin{array}{ccccc}
B_{11} & \cdots & B_{1 m} & \cdots & B_{1 M_{t}} \\
\vdots & \vdots & \vdots & \vdots & \vdots \\
B_{m 1} & \cdots & B_{m m} & \cdots & B_{m M_{t}} \\
\vdots & \vdots & \vdots & \vdots & \vdots \\
B_{M_{t} 1} & \cdots & B_{M_{t} m} & \cdots & B_{M_{t} M_{t}}
\end{array}\right]
$$

where $B_{i j}$ is $N_{r} \times N_{r}$ matrix. Then we get

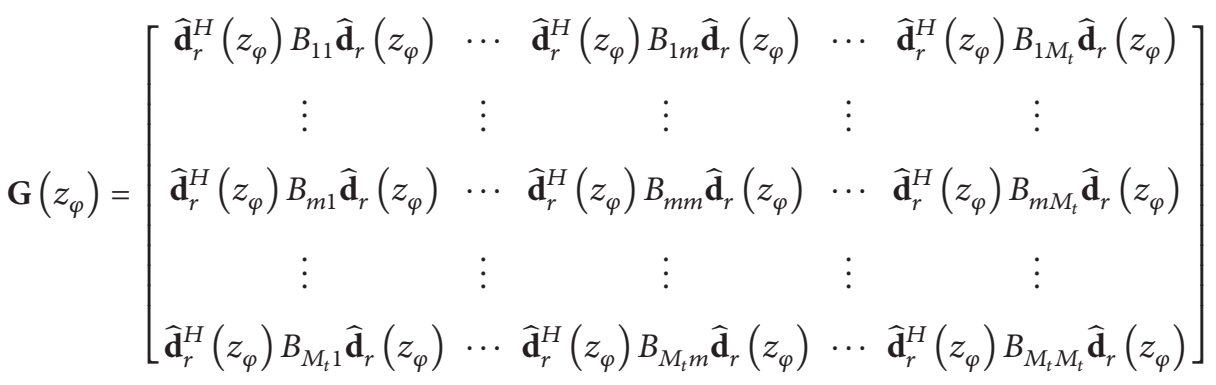

$$
\begin{aligned}
& {\left[\begin{array}{ccccc}
\sum_{\forall p-q=i}\left[B_{11}\right]_{p q} z_{\varphi}^{-i} & \cdots & \sum_{\forall p-q=i}\left[B_{1 m}\right]_{p q} z_{\varphi}^{-i} & \cdots & \sum_{\forall p-q=i}\left[B_{1 M_{t}}\right]_{p q} z_{\varphi}^{-i} \\
\vdots & \vdots & \vdots & \vdots & \vdots \\
\sum_{\forall p-q=i}\left[B_{m 1}\right]_{p q} z_{\varphi}^{-i} & \cdots & \sum_{\forall p-q=i}\left[B_{m m}\right]_{p q} z_{\varphi}^{-i} & \cdots & \sum_{\forall p-q=i}\left[B_{m M_{t}}\right]_{p q} z_{\varphi}^{-i} \\
\vdots & \vdots & \vdots & \vdots & \vdots \\
\sum_{\forall p-q=i}\left[B_{M_{t} 1}\right]_{p q} z_{\varphi}^{-i} & \cdots & \sum_{\forall p-q=i}\left[B_{M_{t} m}\right]_{p q} z_{\varphi}^{-i} & \cdots & \sum_{\forall p-q=i}\left[B_{M_{t} M_{t}}\right]_{p q} z_{\varphi}^{-i}
\end{array}\right] .}
\end{aligned}
$$

The $P$ roots $z_{\varphi}^{(p)}(p=1 \cdots P)$ inside and closest to the unitary circle of the polynomial $\operatorname{det}\left[\mathbf{G}\left(z_{\varphi}\right)\right]$ allow estimating the DOA angles given by $\widehat{\varphi}_{p}=\operatorname{angle}\left(z_{\varphi}^{(p)}\right)(p=1 \cdots P)$. We can see that the computational complexity is burdensome because of the high degree of the polynomial. Here we use a fast rootMUSIC method [17] to reduce the computational complexity. The algorithm, no matter how large the degree of the polynomial is, only needs to calculate $P$ roots (the number of targets).
By substituting the obtained roots $z_{\varphi}^{(p)}(p=1 \cdots P)$ and using (14) in the expression (19), we constitute the following equation:

$$
\begin{aligned}
f\left(\theta, \widehat{\varphi}_{p}\right) & =\widehat{\mathbf{d}}_{t}^{H}(\theta) \widehat{\mathbf{F}}_{t}^{H} \mathbf{G}\left(z_{\varphi}^{(p)}\right) \widehat{\mathbf{F}}_{t} \widehat{\mathbf{d}}_{t}(\theta) \\
& =\widehat{\mathbf{d}}_{t}^{H}\left(z_{\theta}\right) \widehat{\mathbf{F}}_{t}^{H} \mathbf{G}\left(z_{\varphi}^{(p)}\right) \widehat{\mathbf{F}}_{t} \widehat{\mathbf{d}}_{t}\left(z_{\theta}\right) \\
& =\widehat{\mathbf{d}}_{t}^{H}\left(z_{\theta}\right) H\left(z_{\varphi}^{(p)}\right) \widehat{\mathbf{d}}_{t}\left(z_{\theta}\right),
\end{aligned}
$$




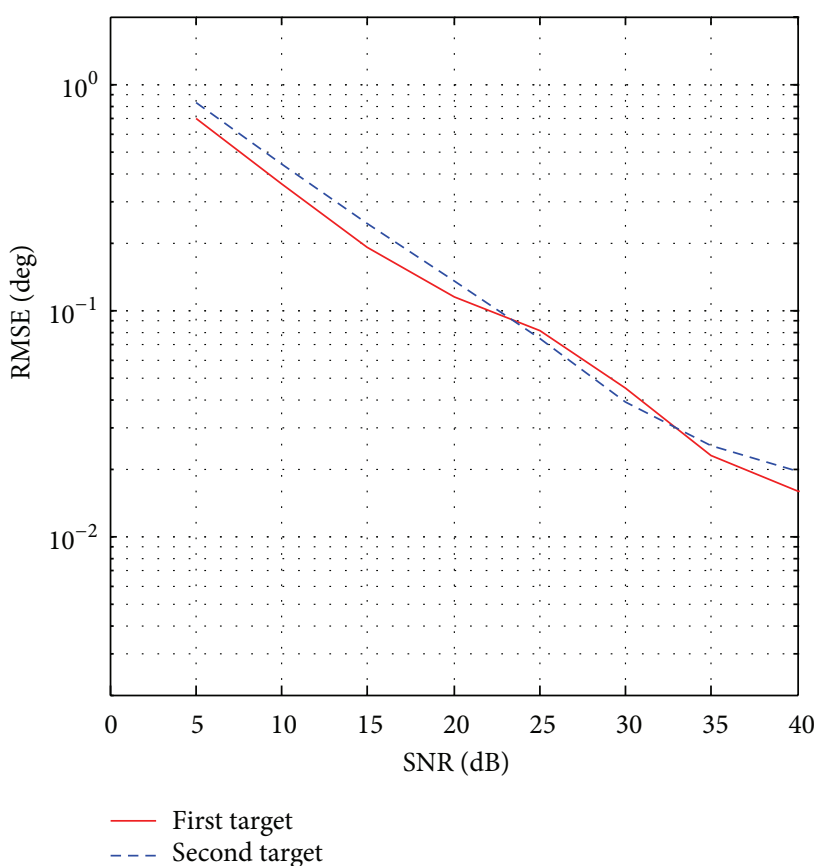

(a) DOA

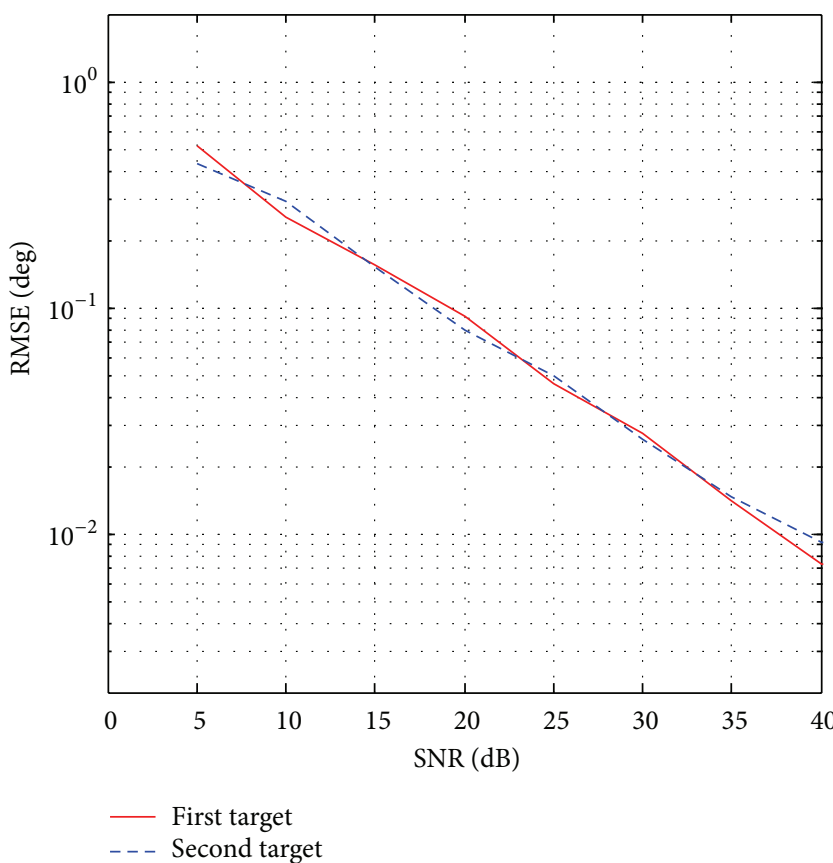

(b) DOD

FIgURE 2: RMSE in DOA and DOD estimation versus SNR.

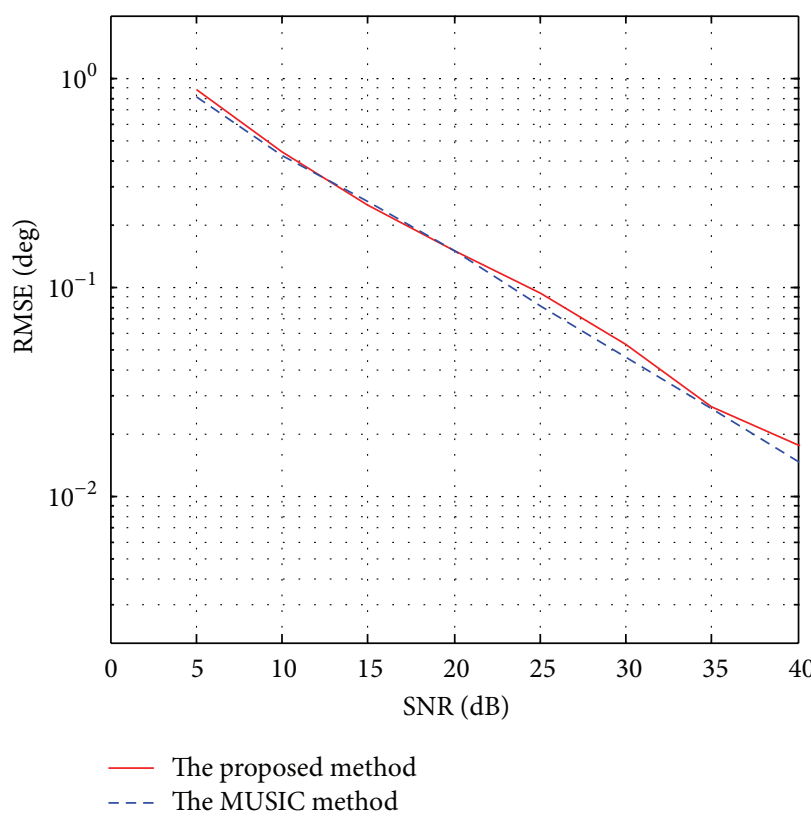

(a) The first target

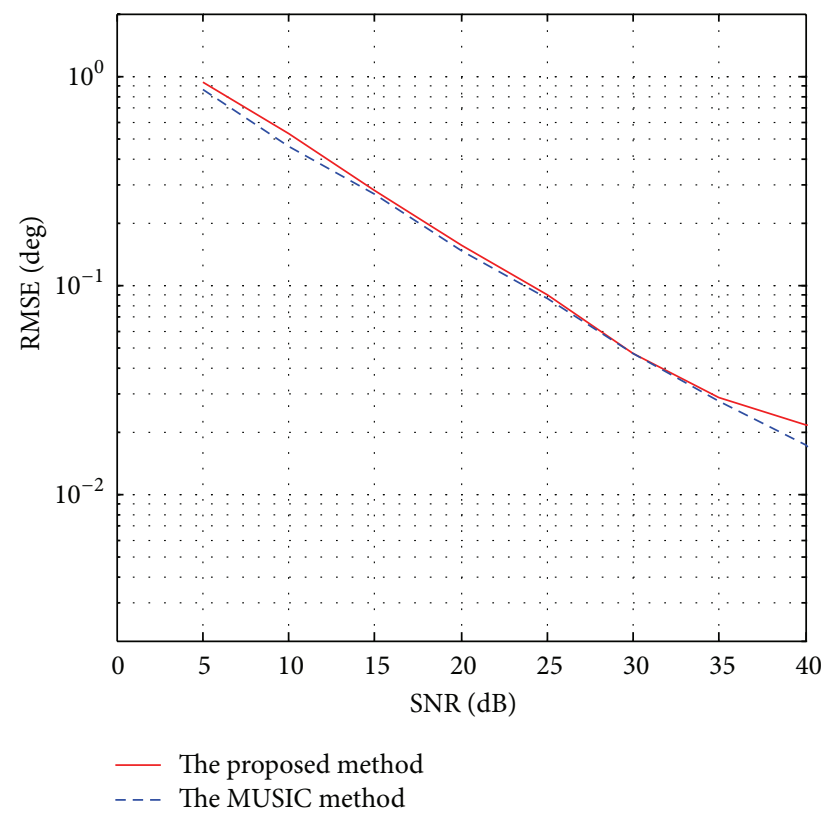

(b) The second target

FIgURE 3: Angle estimation RMSE versus SNR.

where $\mathbf{H}\left(z_{\varphi}^{(p)}\right)=\widehat{\mathbf{F}}_{t}^{H} \mathbf{G}\left(z_{\varphi}^{(p)}\right) \widehat{\mathbf{F}}_{t}$. We can again use the polynomial root technique to get the roots $z_{\varphi}^{(p)}$ with the polynomial (24) equal to zero. The correspondent DOD angle can be given by $\widehat{\varphi}_{p}=\operatorname{angle}\left(z_{\varphi}^{(p)}\right)(p=1 \cdots P)$. Note that the pairing is automatically obtained between the DOA and DOD angles, which avoid the traditional bistatic radar problem of synchronization.

\section{Simulation Results}

Here, we present simulation results showing the statistical performance of the proposed algorithm when using UCA configuration. Consider a narrowband bistatic MIMO radar system with 4 transmit antennas and 3 receive antennas; both are UCAs with radius equal to $\lambda / 4$. For the simulations, the following settings have been used: two uncorrelated sources 
impinging the arrays with equal powers from the angles $\left(\theta_{1}, \varphi_{1}\right)=\left(10^{\circ}, 20^{\circ}\right)$ and $\left(\theta_{2}, \varphi_{2}\right)=\left(50^{\circ}, 40^{\circ}\right), 256$ snapshots, and 100 independent Monte Carlo trials. Figure 1 shows the angle estimation result of two targets for bistatic MIMO radar under the conditions $\mathrm{SNR}=10 \mathrm{~dB}$ and $N_{t}=N_{r}=11$. We can observe that the target directions are well localized and DOA and DOD are automatically paired.

With the same configuration of the simulation as before, Figure 2 shows the DOA and the DOD estimation versus signal noise ratio (SNR) by using the proposed polynomial root finding algorithm. We observe that the proposed algorithm has almost the same performance, no matter if the direction of the target is near or far away from the boresight. It is the advantage of the UCA configuration.

Finally, we compare angle estimation performance of MUSIC spectrum with the searching step $0.01^{\circ}$ and polynomial rooting method with $N_{t}=N_{r}=11$. The root mean squared error (RMSE) of the $p$ th target direction estimation is defined as $\mathrm{RMSE}=\sqrt{E\left(\hat{\theta}_{p}-\theta_{p}\right)^{2}+E\left(\widehat{\varphi}_{p}-\varphi_{p}\right)^{2}}$. Figure 3 demonstrates that the proposed polynomial rooting algorithm provides a similar angle estimation performance to the MUSIC spectral search method with the search interval 0.01 degree, but the MUSIC spectral search method is computationally expensive for such a fine grid search over the whole angle range.

\section{Conclusion}

In this paper, we have proposed a new technique to transform the steering vector of the UCA configuration into a steering vector with a Vandermonde structure in bistatic MIMO radar by using the Jacobi-Anger expansion. The two-step polynomial root finding algorithm has then been used to estimate DOA and DOD of the targets. The simulation results show that the proposed algorithm provides good performances in angle estimation. In addition, the proposed polynomial rooting angle estimation method avoids spectral search and reduces the computational complexity for bistatic MIMO radar with UCA configure.

\section{Acknowledgments}

This work was supported in part by the National Natural Science Foundation of China (61372136, 61172137, and 61271290) and in part by the Fundamental Research Funds for the Central Universities (K5051202005, and K5051302089).

\section{References}

[1] E. Fishler, A. Haimovich, R. S. Blum, L. J. Cimini Jr., D. Chizhik, and R. A. Valenzuela, "Spatial diversity in radars-models and detection performance," IEEE Transactions on Signal Processing, vol. 54, no. 3, pp. 823-838, 2006.

[2] L. Jian, P. Stoica, X. Luzhou et al., "On parameter identifiability of MIMO radar," IEEE Signal Processing Letters, vol. 14, no. 12, pp. 968-971, 2007.

[3] C. Duofang, C. Baixiao, and Q. Guodong, "Angle estimation using ESPRIT in MIMO radar," Electronics Letters, vol. 44, no. 12, pp. 770-771, 2008.
[4] J. Ming, L. Guisheng, and L. Jun, "Joint DOD and DOA estimation for bistatic MIMO radar," Signal Processing, vol. 89, no. 2, pp. 244-251, 2009.

[5] Q. Y. Yin, R. W. Newcomb, and L. H. Zou, "Estimating 2-D angles of arrival via two parallel linear arrays," in Proceedings of the IEEE International Conference on Acoustics, Speech, and Signal Processing, pp. 2803-2806, College Park, Md, USA, May 1989.

[6] N. Liu, L. R. Zhang, J. Zhang et al., "Direction finding of MIMO radar through ESPRIT and Kalman filter," Electronics Letters, vol. 45, no. 17, pp. 908-910, 2009.

[7] C. Yunhe, "Joint estimation of angle and Doppler frequency for bistatic MIMO radar," Electronics Letters, vol. 46, no. 2, pp. 170$172,2010$.

[8] R. Xie, Z. Liu, and Z.-J. Zhang, "DOA estimation for monostatic MIMO radar using polynomial rooting," Signal Processing, vol. 90, no. 12, pp. 3284-3288, 2010.

[9] M. L. Bencheikh, Y. Wang, and H. He, "Polynomial root finding technique for joint DOA DOD estimation in bistatic MIMO radar," Signal Processing, vol. 90, no. 9, pp. 2723-2730, 2010.

[10] Y. Cao, Z. Zhang, F. Dai et al., "Direction of arrival estimation for monostatic multiple-input multiple-output radar with arbitrary array structures," IET Radar, Sonar \& Navigation, vol. 6, no. 7, pp. 679-686, 2012.

[11] A. H. Tewfik and W. Hong, "On the application of uniform linear array bearing estimation techniques to uniform circular arrays," IEEE Transactions on Signal Processing, vol. 40, no. 4, pp. 1008-1011, 1992.

[12] C. P. Mathews and M. D. Zoltowski, "Eigenstructure techniques for 2-D angle estimation with uniform circular arrays," IEEE Transactions on Signal Processing, vol. 42, no. 9, pp. 2395-2407, 1994.

[13] F. Belloni and V. Koivunen, "Beamspace transform for UCA: error analysis and bias reduction," IEEE Transactions on Signal Processing, vol. 54, no. 8, pp. 3078-3089, 2006.

[14] R. Goossens, H. Rogier, and S. Werbrouck, "UCA root-MUSIC with sparse uniform circular arrays," IEEE Transactions on Signal Processing, vol. 56, no. 8, pp. 4095-4099, 2008.

[15] B. D. Rao and K. V. S. Hari, "Performance analysis of rootMUSIC," IEEE Transactions on Acoustics, Speech, and Signal Processing, vol. 37, no. 12, pp. 1939-1949, 1989.

[16] B. Friedlander, "The root-MUSIC algorithm for direction finding with interpolated arrays," Signal Processing, vol. 30, no. 1, pp. 15-29, 1993.

[17] J. Zhuang, W. Li, and A. Manikas, "Fast root-MUSIC for arbitrary arrays," Electronics Letters, vol. 46, no. 2, pp. 174-176, 2010.

[18] F. Belloni, A. Richter, and V. Koivunen, "DoA estimation via manifold separation for arbitrary array structures," IEEE Transactions on Signal Processing, vol. 55, no. 10, pp. 4800-4810, 2007.

[19] M. Rubsamen and A. B. Gershman, "Direction-of-arrival estimation for nonuniform sensor arrays: from manifold separation to Fourier domain MUSIC methods," IEEE Transactions on Signal Processing, vol. 57, no. 2, pp. 588-599, 2009.

[20] M. A. Doron and E. Doron, "Wavefield modeling and array processing, part I-spatial sampling," IEEE Transactions on Signal Processing, vol. 42, no. 10, pp. 2549-2559, 1994. 

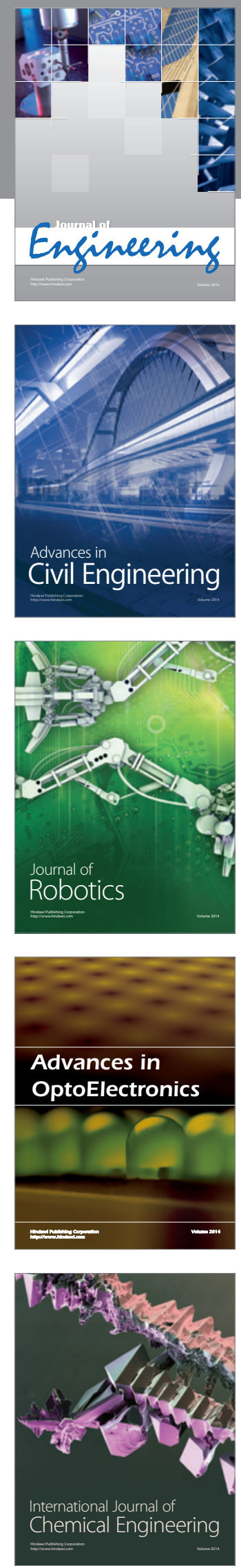

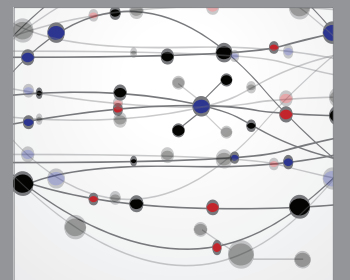

The Scientific World Journal
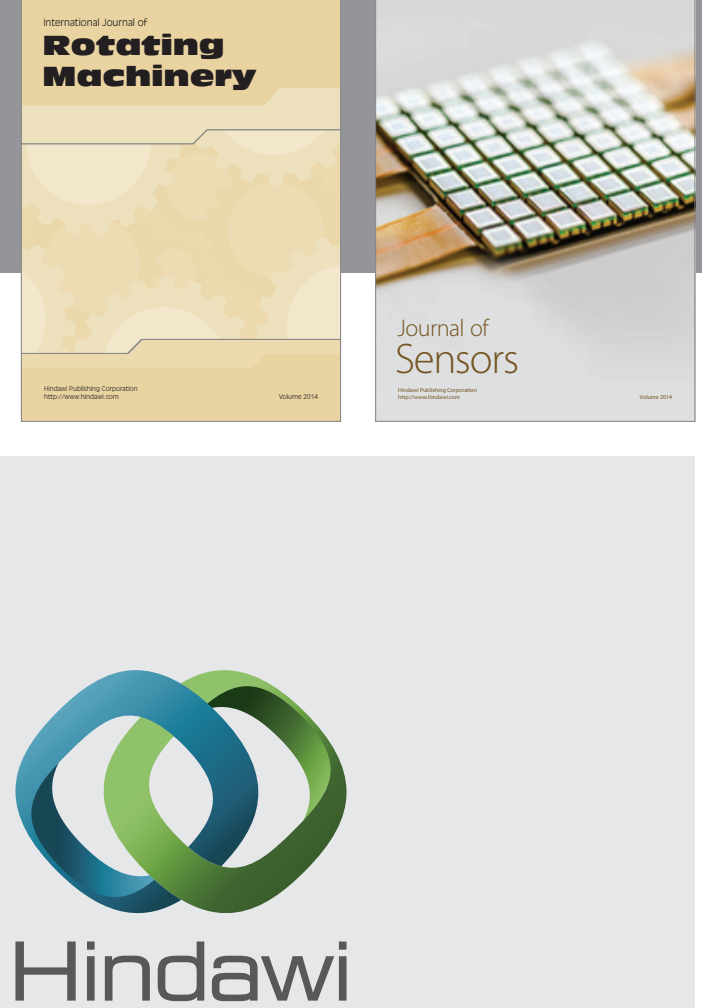

Submit your manuscripts at http://www.hindawi.com
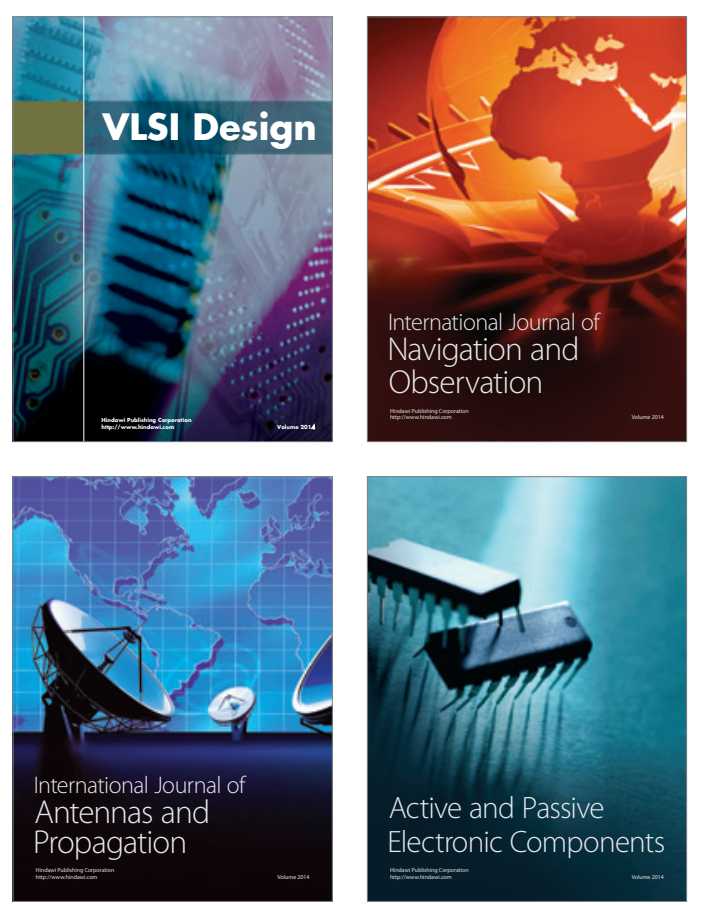
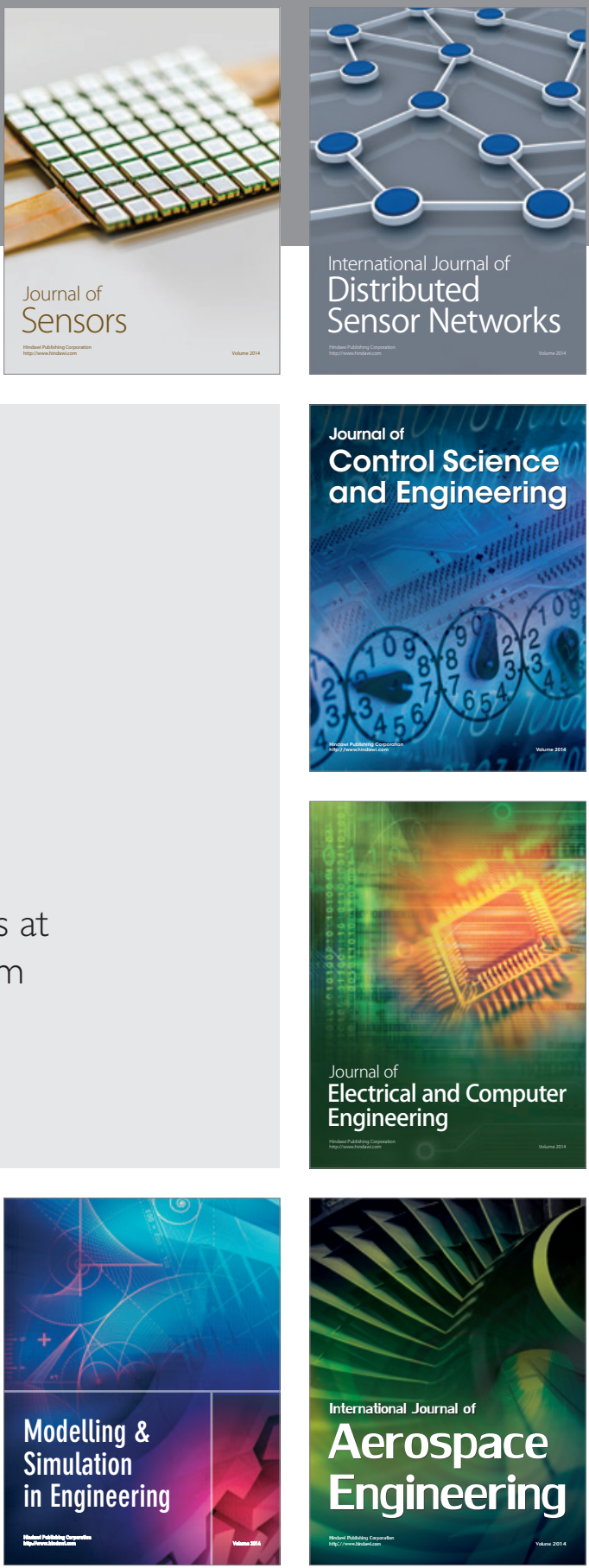

Journal of

Control Science

and Engineering
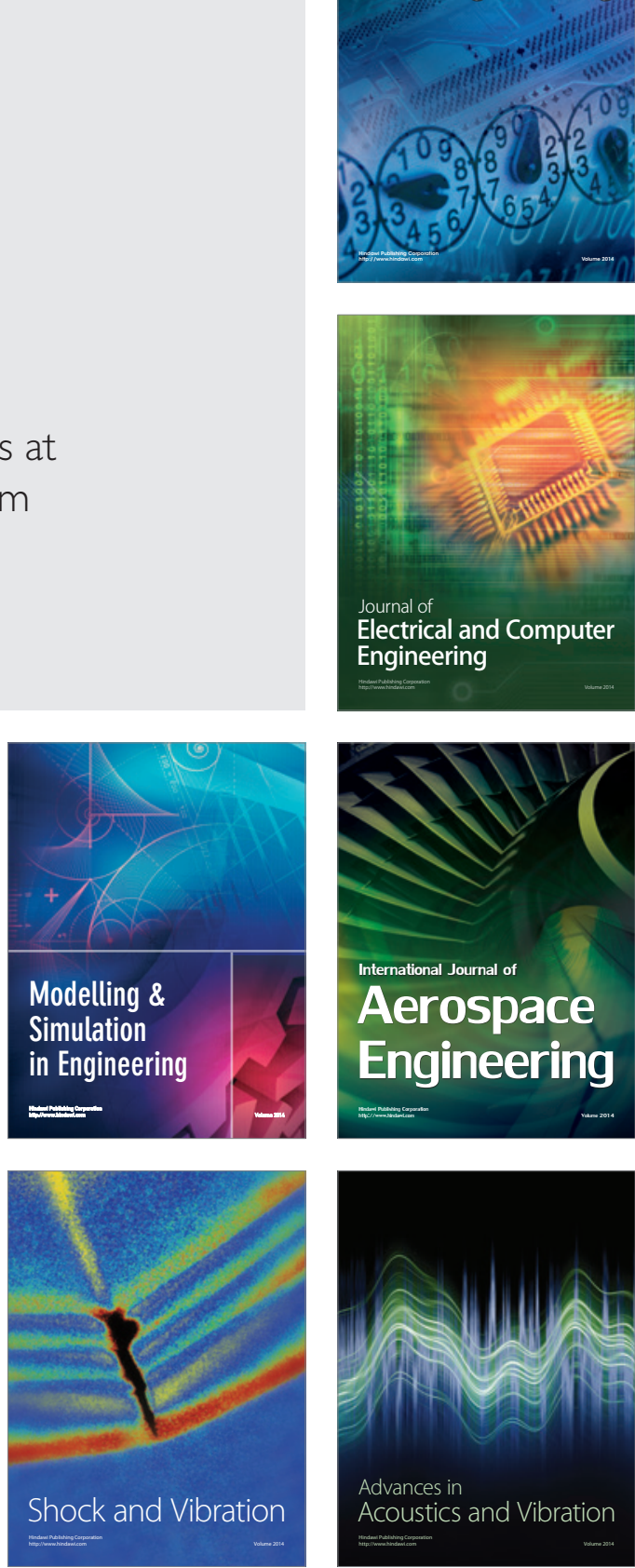